Uniqueness, Shape, and Dimension in EIT

Lionheart, William R

1999

MIMS EPrint: 2015.48

Manchester Institute for Mathematical Sciences

School of Mathematics

The University of Manchester

\footnotetext{
Reports available from: http://eprints.maths.manchester.ac.uk/

And by contacting: The MIMS Secretary

School of Mathematics

The University of Manchester

Manchester, M13 9PL, UK
}

ISSN 1749-9097 


\title{
Uniqueness, Shape, and Dimension in EIT
}

\author{
WILLIAM R. B. LIONHEART \\ Electrical Impedance Tomography Research Group, School of Computing and \\ Mathematical Sciences, Oxford Brookes University, Oxford OX3 OBP, United Kingdom
}

\begin{abstract}
We briefly review the known mathematical results on uniqueness of solution in electrical impedance tomography (EIT). Generally, a real or complex conductivity is determined uniquely by complete boundary data. Uniqueness results are also known for planar resistor networks. However, it is common to make gross errors in the forward modeling of the electrical fields and this may result in no consistent solution. In particular, a two-dimensional model is often used when data are collected from a three-dimensional domain. The boundary shape is often inaccurately known, and commonly modeled by a circle. No model conductivity consistent with measured data exists when the dimension or the boundary shape is wrong.
\end{abstract}

\section{UNIQUENESS OF SOLUTION}

Electrical impedance tomography seeks to recover the interior conductivity from measurements of current and voltage on the bourdary of a body. It is important to ask from the start if the inverse problem of recovering the unknown conductivity from boundary data has a unique solution. This has been an active area of mathematical research since the early 1980s. There are many useful results as well as some unanswered questions. For the direct current case, Maxwell's equations reduce to

$$
\nabla \cdot(\sigma \nabla \phi)=0
$$

which is the continuum version of Kirchhoff's and Ohm's law. Suppose the potential $\phi$ is known on the boundary for every possible pattern of injected current $j=\sigma \partial \phi / \partial \mathbf{n}$ (here, $\mathbf{n}$ is the unit outward normal to the boundary). There is only one conductivity $\sigma(x, y, z)$ that results in this complete set of boundary data, provided that the conductivity satisfies certain smoothness conditions. Most of the known uniqueness results are summarized by Isakov, ${ }^{1}$ to whom we refer the reader for uniqueness results quoted without references. For the three-dimensional case, a conductivity with at least two bounded derivatives is uniquely determined by complete data. In the two-dimensional case, two derivatives (in the generalized sense) that are square-integrable are sufficient. Less is known about the more realistic case of discontinuous conductivities; however, in the case where the conductivity is piecewise analytic (possibly discontinuous on analytic curves), uniqueness holds.

If only the internal boundaries of a piecewise constant conductivity need to be recovered, there are some useful results in the two-dimensional case. In particular, certain simply connected domains can be uniquely identified by a single set of boundary voltage and current data. For recent results on reconstructing unknown internal boundaries, see reference 2 .

Of course, real EIT systems use a finite number of electrodes to inject current and measure voltage. Very little is known about uniqueness in this case, although it is obvious that one cannot recover more unknown parameters of the conductivity than the number of independent measurements. If one models the body by a planar resistor network with suf- 
ficient connections and only $N(N-1) / 2$ resistors, where $N$ is the number of electrodes, then it is known that the resistor values, and the topology of the resistor network up to stardelta transformations, can be determined from current and voltage measurements at these electrodes. ${ }^{3}$ In the bioimpedance case, we would prefer this result to extend to RC networks, but it is not known if that is the case.

When an alternating current is used, neglecting transient effects, the time-harmonic Maxwell's equations can be assumed to apply:

$$
\begin{gathered}
\nabla \times \mathbf{E}=j \omega \mu \mathbf{H} \\
\nabla \times \mathbf{H}=-j(\sigma+j \omega \varepsilon) \mathbf{E} .
\end{gathered}
$$

In this case, we have three unknown inhomogeneous scalar fields $-\sigma, \varepsilon$, and $\mu$. If these are three times differentiable, then Ola et al. ${ }^{4}$ have proved that they are uniquely determined from complete measurements of tangential electric and magnetic fields at the boundary, for a single fixed (nonresonant) frequency $\omega$. However, in the bioimpedance case at sufficiently low frequencies, we can neglect $\omega \mu$, in which case we have the same equation as the direct current case with the conductivity replaced by the complex admittivity $\sigma+j \omega \varepsilon$. In this case, a complete knowledge of the complex current and voltage data at the boundary is sufficient to uniquely determine the admittivity.

\section{THE ANISOTROPIC CASE}

Biological tissue often has anisotropic electrical properties. In this case, the conductivity is replaced by a symmetric matrix. This means that we have six unknowns- $\sigma_{11}, \sigma_{22}$, $\sigma_{33}, \sigma_{12}, \sigma_{23}$, and $\sigma_{13}$-at each point. In this case, the conductivity matrix is not uniquely determined by boundary data. It is easy to construct two matrix-valued conductivity functions that give rise to the same data-one simply chooses them to be related by a coordinate change that preserves coordinates on the boundary. One cannot tell if the conductivity in the interior has been distorted, provided that distortion does not change the boundary. At least for analytical conductivities in three dimensions, this is the only ambiguity and we can recover the six unknown functions with an ambiguity of three functions. In two dimensions, essentially the same result is true for conductivities that are three times differentiable. Furthermore, if accurate in vivo conductivity measurements of anisotropic tissue are required, one must obtain information in addition to the boundary electrical measurements. One option is to estimate the principal directions of the conductivity matrix at each point using anatomical data, such as the alignment of muscle fibers, from magnetic resonance images. Some uniqueness results for the anisotropic uniqueness problem with additional data are given in reference 5. No results are known for the anisotropic full Maxwell's equations.

\section{MODELING ERRORS}

In electrical impedance tomography, some forward model must be used to solve for the electrical fields, given some assumed conductivity distribution. This may take the form of an analytical or semianalytical solution where a simple linear algorithm is used with sim- 
ple geometry and a homogeneous background, or a finite element model where more general geometry or an inhomogeneous initial conductivity is used.

Often for reasons of simplicity or speed, a two-dimensional forward model is used for a three-dimensional domain. Similarly, a simple circular geometry is often assumed for the boundary when in fact the boundary has an irregular shape.

A reconstruction algorithm attempts to find a conductivity in the forward model that best fits the measured current and voltage data. If the model is wrong, it is interesting to know if there is some model conductivity that will exactly match the measured data. If there is not, then the algorithm will not converge; if there is, it may converge to a misleading result. Usually, the former case would be preferable.

\section{ERRORS IN DIMENSION}

\section{Zero Height Electrodes}

Let us suppose that we collect EIT data on some three-dimensional body, but restrict our measurements to some plane. Let us make the idealized assumption that our current drive electrodes are points and we can make point voltage measurements on the specified plane. If a point source of current is applied to the surface of a three-dimensional domain, even one of smoothly varying conductivity, the resulting potential will be of order $1 / r$ as the distance $r$ from the source tends to zero. By contrast, a point source applied to the surface of a two-dimensional domain will result in a potential with a singularity of order $\log r$. We see from this argument that it is possible to determine if one has a two- or a threedimensional domain provided that one can measure sufficiently close to a drive electrode. Consequently, one cannot find a two-dimensional model conductivity consistent with the data collected from a three-dimensional domain with zero height electrodes.

\section{The Separable Case}

If the conductivity separates in some coordinate system, such as $\sigma(x, y, z)=s(x, y) \zeta(z)$, then it is possible to use the separation of variables in the solution of the forward problem $\nabla \cdot \sigma \nabla \phi=0$. The particular case of $\zeta=1$ of the translation invariant conductivity was analyzed by Ider et al. ${ }^{6}$ Let us assume for simplicity, following reference 6 , that the domain is the cylinder $x^{2}+y^{2} \leq R^{2},-a \leq z \leq a$. Measurements will be made on the plane $z=0$.

It is easy to check that the potential has the form

$$
\phi(x, y, z)=\sum_{k=0}^{\infty} V_{k}(x, y) \cos \left(\frac{k \pi}{a}\right) z
$$

where each $V_{k}$ satisfies the two-dimensional partial differential equation

$$
\nabla \cdot s \nabla V_{k}-s(k \pi / a)^{2} V_{k}=0
$$

Now suppose that the current density on the boundary satisfies $J(\theta, z)=f(\theta) g(z)$, where

$$
g(z)=\sum_{k=0}^{\infty} c_{k} \cos \left(\frac{k \pi}{a}\right) z .
$$


Then, the boundary condition for $V_{k}$ is

$$
s \frac{\partial V_{k}}{\partial n}=c_{k} f(\theta)
$$

Suppose that we apply our current using electrodes of height $h$ so that $g(z)=1 / h$ for $|z|<h / 2$ and $g(z)=0$ elsewhere. Hence,

$$
c_{0}=h / a \quad \text { and } \quad c_{k}=\left(\frac{2}{k h \pi}\right) \sin \left(\frac{k \pi h}{2 a}\right) \quad \text { for } \quad k>0 .
$$

In the extreme case, $h=a$ electrodes run the full height of the cylinder and we have a genuinely two-dimensional problem. Here, $V_{k}=0$, except for $k=0$, and we can solve the usual conductivity equation for two dimensions. As $h / a$ becomes smaller, the series (3) for $\phi$ converges more slowly and we will have to solve more two-dimensional forward problems (4) to get the same accuracy. In the other extreme case where $h \rightarrow 0$, we have $g(z)=\delta(z) / a$ and $c_{k}=1 / a$. Here, the series (3) for $\phi$ is divergent at $z=0$ because the potential will generally have a $\log |z|$ singularity where $f(\theta) \neq 0$.

This analysis gives a clue as to why EIT reconstruction algorithms that use two-dimensional models of three-dimensional domains work to some extent. If the conductivity is approximately translation invariant (this is only a very crude approximation in the thorax for example, but perhaps better on a limb), then the error in assuming a two-dimensional model is the error in truncating the series (3) at the first term. This error diminishes as the height of the electrode increases.

As yet, no uniqueness results are known for the recovery of a translationally invariant conductivity from EIT data in the plane. However, the inverse problem has been solved numerically by Ider et al. ${ }^{6}$ and by Butler and Bonnecaze. ${ }^{7}$

\section{INCORRECT BOUNDARY SHAPE}

\section{The Two-Dimensional Case}

As we have seen, it is unwise to model a three-dimensional region using a two-dimensional model, unless the conductivity and the current drive electrodes are translationally invariant. Let us suppose that we are in this situation, in which case the model and the body can be assumed to be two-dimensional. We will also assume that the conductivity is isotropic.

The two-dimensional case is special in that there is a rich variety of conformal mappings that can transform any smooth simply connected domain to any other. Conformal mappings are smooth invertible transformations that preserve angle, but not length. Suppose that $\sigma$ and $\phi$ are conductivity and potential fields satisfying the two-dimensional conductivity equation $\nabla \cdot \sigma \nabla \phi=0$. A conformal mapping transforms $\sigma \mapsto \tilde{\sigma}$ and $\phi \mapsto \tilde{\phi}$, where $\nabla \cdot \tilde{\sigma} \nabla \tilde{\phi}=0$. The boundary current density $\sigma(\partial \phi / \partial \mathbf{n}) d s$, where $s$ is the arc length coordinate on the boundary of the domain, is invariant under conformal mappings.

Suppose now that we do not know the shape of the boundary, but we measure all current and voltage data with respect to some coordinate on the boundary. This corresponds roughly to knowing the electrode numbering, but not their spacing. To make the current measurement independent of the length scale, we assume that the cumulative current 


$$
I(s)=\int_{0}^{s} \sigma \partial \phi / \partial \mathbf{n} d s
$$

is known together with the corresponding potential $\phi(s)$. Assuming some shape for the boundary, suppose that we reconstruct a conductivity consistent with these data. If we apply any conformal mapping to this domain and conductivity, the result will still be consistent with the measured data. As a consequence, we would expect to be able to reconstruct a consistent conductivity using the wrong boundary shape, and our image would be a conformal distortion of the true image.

However, this model of measurement may be unrealistic. For example, if the length scale on the boundary were known-imagine, for example, the electrodes were placed with the aid of a tape measure - then these data would not be invariant under conformal mappings, except for rotations and translations. In this case, only a model with the correct boundary shape and size would fit the measured data.

\section{The Three-Dimensional Case}

Conformal mappings are well known in two dimensions from complex analysis. Among the well-known conformal mappings are the Möbius transformations that correspond to inversions with respect to a circle. In three-dimensional space, the situation is very different. The only conformal mappings that are not similarities (combinations of translations, rotations, reflections, and dilations) are the three-dimensional Möbius transformations. Like their counterparts in two dimensions, these Möbius transformations preserve parts of circles and, of course, parts of spheres too. Here, we treat straight lines and planes as circles and spheres of infinite radius. See Figure 1 for an example.

For an isotropic three-dimensional body, boundary potential and current are invariant under conformal mappings. As in the two-dimensional case, this is the only ambiguity in the boundary shape.

Hence, the shape of our model must be related to the true boundary shape by a conformal mapping. Unlike the two-dimensional case where any two simply connected domains are so related, this is quite a rare occurrence in three dimensions. The set of conformal mappings can be described by 10 parameters. Of these, 7 come from the group of similari-
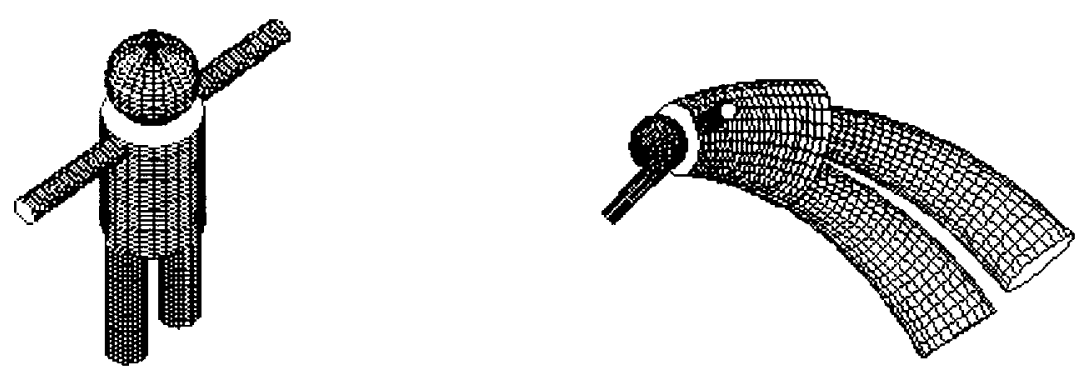

FIGURE 1. A Möbius transformation applied to a simple geometric figure. Note the preservation of circles and spheres. 
ties, which simply means that one cannot determine position, orientation, and overall size by EIT measurement. Three suitably chosen measurements of the geometry of the boundary together with complete EIT data determine both the shape and the conductivity of the body. An example of such a set of measurements is found in reference 8 , where further mathematical details of the above arguments are given.

Therefore, it can be seen that EIT data contain information about both the shape and conductivity and that it is unlikely that a consistent conductivity will be found if one gets the shape wrong.

\section{CONCLUSIONS}

The ideal when imaging three-dimensional bodies with irregular shape is to use a three-dimensional model with measured boundary shape. If the model shape is incorrect and the conductivity is isotropic, then there is no consistent conductivity for the model that will fit the data. This will be observed as a large residual voltage error, so any image produced will be known to be unreliable.

If measurements are taken in the plane, and the conductivity has approximate translational invariance perpendicular to the plane, then one must either use very tall drive electrodes if using a two-dimensional model or use the correct forward model as described by Ider $e t a l .{ }^{6}$ If a length scale on the boundary is fixed in the two-dimensional case, then (as in the three-dimensional case) no consistent conductivity can be found when the model has incorrect boundary shape.

\section{REFERENCES}

1. Isakov, V. 1998. Inverse problems for partial differential equations. In Applied Mathematical Sciences, p. 127. Springer Pub. New York.

2. FRANK, H. \& W. RUNDELL. 1998. The determination of a discontinuity in a conductivity from a single boundary measurement. Inverse Probl. 14: 67-82.

3. Colin de Verdiere, Y., I. Gitler \& D. Vertigan. 1996. Reseaux electriques planaires II. Comment. Math. Helv. 71: 144-167.

4. Ola, P., L. Paeivaerinta \& E. Somfrsalo. 1993. An inverse boundary value problem in electrodynamics. Duke Math. J. 70(no. 3): 617-653.

5. LIONHF.ART, W.R.B. 1997. Conformal uniqueness results in anisotropic electrical impedance imaging. Inverse Probl. 13: 125-134.

6. Ider, Y.Z., N.G. Gencer, E. Atalar \& H. Tosun. 1990. Electrical-impedance tomography of translationally uniform cylindrical objects with general cross-sectional boundaries. IEEE Trans. Med. Imaging 9(no. 1): 49-59.

7. BUTLER, J.E. \& R.T. BONNECAZE. 1997. Imaging of particle shear migration with electrical impedance tomography. American Institute of Chemical Engineers Annual Meeting.

8. LIONHEART, W.R.B. 1998. Boundary shape and electrical impedance tomography. Inverse Probl. 14: $139-147$. 